\title{
RESPUESTAS RÁPIDAS A LA PANDEMIA DE COVID-19 A TRAVÉS DE LA CIENCIA Y LA COLABORACIÓN GLOBAL: EL ENSAYO CLÎNICO SOLIDARIDAD
}

\author{
Alonso Soto $\circledast^{1,2, a}$, Dante M. Quiñones-Laveriano ${ }^{10, b}$, Patricia J. Garcia® ${ }^{3, c}$, \\ Eduardo Gotuzzo (1) ${ }^{4, d}$, Ana María Henao-Restrepo (i] ${ }^{5, e}$ \\ 1 Instituto de Investigación en Ciencias Biomédicas, Universidad Ricardo Palma, Lima, Perú. \\ 2 Departamento de Medicina, Hospital Nacional Hipólito Unanue, Lima, Perú. \\ 3 Facultad de Salud Pública y Administración, Universidad Peruana Cayetano Heredia, Lima, Perú. \\ ${ }^{4}$ Instituto de Medicina Tropical Alexander von Humboldt, Universidad Peruana Cayetano Heredia, Lima, Perú. \\ 5 Organización Mundial de la Salud, Ginebra, Suiza. \\ a Médico internista; ${ }^{\mathrm{b}}$ médico cirujano; ${ }^{\mathrm{c}}$ médica cirujana, doctora en Medicina; ${ }^{\mathrm{d}}$ médico especialista en Enfermedades \\ Infecciosas y Tropicales; ${ }^{\mathrm{e}}$ médica epidemióloga
}

\section{RESUMEN}

La COVID-19 representa una crisis global. La realización rápida de un ensayo clínico con la rigurosidad necesaria para obtener resultados confiables requiere la colaboración de diversos actores que participan en el desarrollo, evaluación y autorización de los ensayos clínicos (EC), como el patrocinador del ensayo, los investigadores, la autoridad regulatoria y el comité de ética (CE). Llevar a cabo estos estudios no solo es científicamente apropiado, sino una obligación ética y moral para ofrecer a las personas infectadas con COVID-19 un tratamiento efectivo. Solidaridad es un megaensayo clínico que reclutará miles de sujetos de investigación con enfermedad moderada a grave, a quienes se les asignará aleatoriamente a uno de los grupos de tratamiento en evaluación incluyendo hidroxicloroquina, lopinavir/ritonavir asociado o no a interferón; o remdesivir en comparación con el manejo estándar. El Perú se ha sumado a la lista de países donde se reproducirá el ensayo, mediante el cual se podrá identificar rápidamente si alguno de estos fármacos ofrece un beneficio real a los pacientes.

Palabras clave: COVID-19; SARS-CoV-2; Ensayos Clínicos; Hidroxicloroquina; Remdesivir (fuente: DeCS BIREME).

\section{RAPID RESPONSES TO THE COVID-19 PANDEMIC THROUGH SCIENCE AND GLOBAL COLLABORATION: THE SOLIDARITY CLINICAL TRIAL}

Citar como: Soto A, QuiñonesLaveriano DM, Garcia PJ, Gotuzzo E, Henao-Restrepo AM. Respuestas rápidas a la pandemia de COVID-19 a través de la ciencia y la colaboración global: el ensayo clínico Solidaridad. Rev Peru Med Exp Salud Publica. 2020;37(2):356-60. doi: https://doi. org/10.17843/rpmesp.2020.372.5546

Correspondencia: Alonso Soto; Instituto de Investigación en Ciencias Biomédicas de la Facultad de Medicina Humana de la Universidad Ricardo Palma; avenida Benavides 5440, Santiago de Surco, Lima, Perú; sotosolari@gmail.com

Recibido: $11 / 04 / 2020$ Aprobado: 06/05/2020 En línea: 18/05/2020

\begin{abstract}
COVID-19 represents a global crisis. Rapidly conducting a clinical trial with the rigor necessary to obtain reliable results requires the collaboration of various participants involved in the development, evaluation and authorization of clinical trials (CT) such as the trial sponsor, researchers, regulatory authority and the ethics committee (EC). Carrying out these studies is not only scientifically appropriate, but an ethical and moral obligation to guarantee our patients effective treatment. SOLIDARITY is a mega clinical trial that recruited thousands of subjects with moderate to severe disease, who were randomly assigned to one of the treatment groups under evaluation, including hydroxychloroquine, lopinavir/ritonavir associated or not with interferon; or remdesivir compared to standard therapy. Peru has joined the list of countries where the trial will be reproduced, through which it will be possible to quickly identify if any of these drugs offers a real benefit to patients.
\end{abstract}

Keywords: COVID-19; SARS-CoV-2; Clinical Trials; Hydroxychloroquine; Remdesivir (source: MeSH NLM).

\section{INTRODUCCIÓN}

La pandemia del nuevo coronavirus SARS-CoV-2 es una de las principales crisis humanitarias de la historia reciente, que ha puesto en evidencia la limitada capacidad de respuesta de muchos sistemas de salud ante situaciones extremas, pues estos han colapsado ante la ingente demanda de atención, incluso en países con sistemas de salud que podríamos considerar 
ejemplares ${ }^{(1)}$. La COVID-19 ha afectado ya a más de 4 millones de personas en todo el mundo, y matado a cerca de 280 mil víctimas ${ }^{(2)}$. Esta crisis supera los recursos sanitarios de nuestra sociedad, ha mermado considerablemente la economía mundial y obligado a los gobiernos a invertir enormes cantidades de dinero para su rescate ${ }^{(3)}$, sin mencionar el incalculable costo social y el sufrimiento de las familias de los pacientes afectados.

En las últimas décadas nos hemos enfrentado, con éxito variable, a diversas epidemias, algunas causadas por otros tipos de coronavirus, como el SARS-CoV en 2002 y el MERS$\mathrm{CoV}$ en $2012^{(4)} \mathrm{y}$ a pandemias como la de 2009 , causada por el virus de la influenza A (H1N1), la cual se calcula ocasionó la muerte de 284500 personas en todo el mundo ${ }^{(5)}$. Estas epidemias preocupan en gran medida a gobiernos y direcciones de salud nacionales, que, en la premura de mitigar su impacto en la sociedad, gastan millones de dólares en intervenciones y compras de fármacos. Si bien muchas veces pueden presentarse como promisorios, dichos fármacos no cuentan con evidencia suficiente para mostrar su beneficio, lo cual expone a numerosos pacientes a drogas potencialmente inútiles $\mathrm{o}$, en el peor de los casos, perjudiciales ${ }^{(6)}$. Esto se debe a que muchos médicos y decisores en salud consideran que realizar ensayos clínicos (EC) rigurosos de fármacos en el contexto de una pandemia es, cuanto menos, utópico.

La situación actual no es diferente a las descritas en otros países. Más aún en medio de una pandemia con números crudos y muchísimo más preocupantes, se crea la angustia en los decisores de políticas y en los profesionales sanitarios por presentar algún fármaco contra la enfermedad o proponer esquemas de tratamiento. Tal es así que, pese a que aún no hay evidencias de tratamientos efectivos contra la COVID-19, muchos hospitales ${ }^{(7,8)}$, algunas guías de sociedades científicas ${ }^{(9,10)} \mathrm{e}$, incluso, normativas de las direcciones nacionales de salud ${ }^{(11)}$ abren la posibilidad de usar diversas drogas y combinaciones de drogas.

El Ministerio de Salud (MINSA), en su documento técnico Prevención, Diagnóstico y Tratamiento de personas afectadas por COVID-19 en el Perú, ofrece a los médicos tratantes la opción de usar algunos fármacos, cuya eficacia aún no está demostrada, como la cloroquina y la hidroxicloroquina combinadas o no con azitromicina. Esto se basa en algunas experiencias promisorias y mediáticas, y en la demanda de las sociedades médico-científicas y de los expertos locales. Si bien es cierto que se sugiere la valoración individual del tratamiento, en la práctica se difunden como recomendaciones estándares y, de hecho, se está contemplando el uso de algunos de estos fármacos en la atención primaria, incluso sin un balance claro entre su incierta eficacia y los posibles efectos adversos. El mismo documento técnico menciona claramente que no existe evidencia de calidad que permita sustentar la recomendación de algún tratamiento específico para la COVID-19 por lo cual resulta imperativo generar evidencia de calidad.

\section{¿Por qué es necesario realizar ensayos clínicos para evaluar intervenciones terapéuticas contra la COVID-19?}

La respuesta es simple, porque actualmente no existe un tratamiento efectivo validado para la COVID-19, y decir que «pareciera ser efectivo» no es suficiente para exponer a los pacientes a los posibles riesgos de las drogas. El EC controlado y aleatorizado es considerado el estándar de referencia para evaluar la utilidad de las intervenciones en salud. Dentro de la rigurosidad de su desarrollo se atiende la necesidad, no solo de demostrar la eficacia de un fármaco o una intervención, sino también de proteger la seguridad del sujeto de investigación de posibles efectos adversos. Para ello debe cumplir con requisitos sumamente estrictos y condiciones necesarias para su adecuada ejecución, que establecen las agencias reguladoras de medicamentos de cada uno de los países. Asimismo, resulta necesario resaltar que el desarrollo de cualquier producto en investigación conlleva, por lo general, varios años desde su descubrimiento hasta contar con un registro sanitario y poder ser usado en las condiciones objeto de estudio y formar parte de la práctica clínica rutinaria. Desde luego esto no es aplicable a situaciones de pandemia, como la que actualmente vivimos.

Así pues, la única manera de conocer el efecto real de una intervención es a través de la comparación aleatorizada con el manejo estándar o, en caso no exista uno, con un placebo. Por otro lado, es indispensable que exista un número adecuado de participantes en cada grupo de intervención, que permita una potencia estadística suficiente para encontrar las diferencias entre los grupos de tratamiento y que, asimismo, prevenga diferencias espurias debido al azar (error tipo alfa), las que ciertamente tendrán más posibilidades de ser publicadas en desmedro de aquellos estudios que no encuentren diferencias significativas, lo cual se conoce como sesgo de publicación.

Algunos de los fármacos propuestos contra la COVID-19 fueron sometidos a ensayos pequeños, de bajo poder estadístico y con criterios de selección poco específicos que impidieron la extrapolación de sus resultados a la gran mayoría de pacientes ${ }^{(12)}$, o, en su defecto, tuvieron sesgos importantes, como la ausencia de aleatorización o de controles adecuados. Uno de los estudios más publicitados y base de muchas recomendaciones ha sido cuestionado por la propia International Society of Antimicrobial Chemotherapy ${ }^{(13)}$, por falta de estándares científicos apropiados ${ }^{(14)}$. Estas investigaciones nos dejan expectantes por ver si cualquiera de estos fármacos podría o no funcionar, pero deben corroborarse con un estudio que pueda superar las limitaciones antes presentadas.

La realización rápida de un EC con la rigurosidad necesaria para obtener resultados confiables en una situación apremiante requiere la colaboración de muchos centros de investigación a nivel mundial, por lo cual urge un acuerdo multinacional para reclutar la mayor cantidad de sujetos de 
investigación en el tiempo más corto posible, viabilizar los procedimientos operativos y afianzar el compromiso de los gobiernos en formar parte de esta alianza. La necesidad de adaptar y agilizar los procedimientos de revisión sin dejar de lado la protección del sujeto de investigación es un imperativo reconocido globalmente ${ }^{(15)}$. La Organización Mundial de la Salud (OMS) recomienda contar con procedimientos coordinados para revisar estudios multicéntricos que agilicen las evaluaciones y eviten la duplicación de esfuerzo, ya sea nacional o internacionalmente ${ }^{(16)}$.

Es de suma importancia tener en claro que realizar estos estudios no solo es científicamente apropiado, sino una obligación ética y moral para garantizar a los pacientes un tratamiento efectivo.

\section{El ensayo clínico Solidaridad}

El 20 de marzo, la OMS anunció el desarrollo de la iniciativa Solidaridad, impulso coordinado con varios países para realizar un megaensayo clínico que permita reclutar miles de sujetos de investigación en diversos centros de investigación alrededor del mundo, los cuales serán asignados aleatoriamente a uno de los grupos de tratamiento en evaluación o, en su defecto, al grupo de tratamiento estándar local, el cual consiste en soporte vital. El estudio se focaliza en quienes tengan la COVID-19 de moderada a grave, ya que se busca también poder disminuir la proporción de pacientes que necesiten cuidados intensivos.

Con el fin de obtener evidencia que pueda ser extrapolable a la práctica habitual, se hace un ensayo pragmático en condiciones reales, sin la limitada validez externa que podrían tener los ensayos clásicos y con desenlaces sencillos de registrar mediante tecnologías de información, como el tiempo de hospitalización, la fecha de alta o de defunción, o el requerimiento de ventilación mecánica; facilitando así el trabajo de médicos que probablemente tengan una excesiva carga laboral. La asignación de los sujetos de investigación, todos mayores de edad, a los grupos de tratamiento o al grupo control es de forma aleatoria mediante un programa al que los médicos tendrán acceso de forma digital. Se registran las comorbilidades, como diabetes, enfermedad cardíaca, enfermedad pulmonar crónica, enfermedad hepática crónica, asma, VIH-SIDA o tuberculosis. Los medicamentos en evaluación fueron seleccionados por su potencial beneficio de acuerdo con los estudios realizados hasta la fecha, y se consideraron aquellos con disponibilidad suficiente para administrarse en una gran cantidad de pacientes ${ }^{(17)}$. Los fármacos propuestos son los siguientes:

Hidroxicloroquina: Esta droga ha mostrado actividad antiviral contra una serie de virus, incluso el Ébola, aunque al probarlo contra este agente, no fue clínicamente efectiva. También ha mostrado actividad inhibitoria contra el SARS-CoV-2 in vitro ${ }^{(18,19)} \mathrm{y}$, como se mencionó anteriormente, hay algunos estudios que reportan mejoras asociadas a su uso ${ }^{(14,20)} \mathrm{y}$ otros no. Se trata de un fármaco con un perfil de seguridad conocido, sin embargo, es necesario tener precaución con su uso, dado que no se conocen las dosis terapéuticas contra la COVID-19 y, además, puede tener efectos adversos, como arritmias y problemas gastrointestinales ${ }^{(21)}$.

Remdesivir: Antiviral desarrollado originalmente para combatir el virus del Ébola ${ }^{(22)}$. Ha demostrado actividad in vitro contra diversos tipos de coronavirus, incluso el SARS-CoV y el MERSCoV (23) y, recientemente, también contra el SARS-CoV-2 ${ }^{(19)}$. Si bien los reportes de su beneficio frente a la COVID-19 de momento se limitan a pequeños reportes ${ }^{(24)}$, estos son promisorios. Además, existen datos acerca de su seguridad en humanos ${ }^{(25)}$.

Lopinavir/ritonavir: El lopinavir es un antiviral del tipo de inhibidores de proteasas usado con el ritonavir para tratar el VIH. El lopinavir ha demostrado actividad in vitro contra el SARS-CoV-2 ${ }^{(26)}$ y su uso combinado ha podido detener la progresión de la infección por MERS-CoV en monos titíes ${ }^{(27)}$. En un reciente ensayo abierto con 199 participantes se descubrió que el tratamiento con lopinavir/ritonavir redujo en 5,8\% la mortalidad frente al tratamiento convencional (solo soporte) aunque esta diferencia no tuvo significancia estadística ${ }^{(12)}$.

Lopinavir/ritonavir + interferón beta-1a: El interferón beta-1a es una citoquina con funciones inmuno moduladoras, usado normalmente para tratar la esclerosis múltiple ${ }^{(28)}$. En un estudio en primates, ha demostrado tener efectos beneficiosos en la infección por MERS-CoV ${ }^{(27)}$. Así pues, la adición del interferón beta-1a sobre el tratamiento con lopinavir/ritonavir podría tener un efecto sinérgico sobre los coronavirus ${ }^{(29)}$.

Un aspecto relevante del ensayo Solidaridad, además de la obtención de resultados rápidos, es su naturaleza adaptativa, una metodología relativamente reciente en EC que permite modificar decisiones «sobre la marcha». Esto implica que los resultados serán evaluados frecuentemente por un grupo de expertos, lo cual permitirá tomar decisiones rápidas acerca de la eliminación de alguno de los tratamientos experimentales y su reemplazo por otros fármacos, previa evaluación de su potencial ${ }^{(30)}$.

\section{La colaboración peruana}

El Perú ha mostrado, al igual que numerosos países, la voluntad de colaborar con este ensayo. El 28 de marzo, el ministro de Salud Víctor Zamora envió una carta a Tedros Adhanom, presidente de la OMS, para confirmar la participación del Perú en el ensayo Solidaridad ${ }^{(31)}$, y en 16 de abril la Oficina General de Investigación y Trasferencia Tecnológica (OGITT) del Instituto Nacional de Salud autorizó la ejecución de dicho ensayo en el país. Esto permite no solamente participar en esta carrera multinacional para enfrentar la COVID-19, sino también contribuir con la generación de evidencia 
local respecto al uso de estos tratamientos en estudio, a las situaciones en los que podrían usarse, los cuidados necesarios para los pacientes que los reciben y, así, mejorar la calidad de evidencia científica disponible. Por otro lado, participar en este ensayo asegura la accesibilidad a medicamentos que podrían beneficiar a estos pacientes. Además, considerando que alrededor del $5 \%{ }^{(32)}$ de los pacientes infectados podrían requerir cuidados intensivos, la posibilidad de que alguna estas intervenciones resulte útil contribuiría a evitar el colapso del sistema de salud.

Nuestro país ha participado exitosamente en anteriores estudios de colaboración internacional ${ }^{(33)}$. Hasta el momento estamos autorizados para ejecutar este EC en diversos centros de investigación del Perú, hemos iniciado el reclutamiento de participantes tanto en el sector público como en el privado y ha quedado abierta la posibilidad de ampliar su ejecución en otros centros a fin de alcanzar la cuota de reclutamiento asignada al país.

\section{CONCLUSIÓN}

Estamos en un momento en el cual se requieren respuestas rápidas y urgentes frente a la epidemia del coronavirus. Solidaridad es una colaboración global que permitirá comparar la eficacia de los principales tratamientos que han mostrado un potencial promisorio. La naturaleza pragmática y adaptativa del estudio permitirá obtener resultados rápidos, confiables y aplicables a nuestra población. Hoy más que nunca es el momento de tener evidencia sólida que permita intervenciones eficaces.

\section{REFERENCIAS BIBLIOGRÁFICAS}

1. Tanne JH, Hayasaki E, Zastrow M, Pulla P, Smith P, Rada AG. Covid-19: how doctors and healthcare systems are tackling coronavirus worldwide. BMJ 2020;368:m1090. doi: 10.1136/bmj.m1090.

2. World Health Organization. Coronavirus disease 2019 (COVID-19) situation report - 114 n.d. (acceso 8 abril 2020). Disponible en: https://www.who.int/docs/default-source/coronaviruse/situation-reports/20200513-covid-19-sitrep-114.pdf?sfvrsn=17ebbbe_4.

3. Fernandes N. Economic Effects of Coronavirus Outbreak (COVID-19) on the World Economy. Rochester, NY: Social Science Research Network; 2020. doi: 10.2139/ssrn.3557504.

4. Lee PI, Hsueh PR. Emerging threats from zoonotic coronaviruses-from SARS and MERS to 2019-nCoV. J Microbiol Immunol Infect. 2020; pii: S1684-1182(20)30011-6. doi: 10.1016/j.jmii.2020.02.001.

5. Dawood FS, Iuliano AD, Reed C, Meltzer MI, Shay DK, Cheng PY, et al. Estimated global mortality associated with the first 12 months of 2009 pandemic influenza A H1N1 virus circulation: a modelling study. Lancet Infect Dis 2012;12:687-95. doi: 10.1016/S1473-3099(12)70121-4.

6. Vásquez Cubas H. Controversia sobre oseltamivir y su efectividad contra influenza. An Fac Med 2019;80:234-8. doi: 10.15381/anales.802.16420.

7. Li T. Diagnosis and clinical management of severe acute respiratory syndrome Coronavirus 2 (SARS-CoV-2) infection: an operational recommendation of Peking Union Medical College Hospital (V2.0). Emerg Microbes Infect 2020;9:582-5. doi: 10.1080/22221751.2020.1735265.

8. Nicastri E, Petrosillo N, Bartoli TA, Lepore L, Mondi A, Palmieri F, et al. National Institute for the Infectious Diseases "L. Spallanzani", IRCCS. Recommendations for COVID-19 clinical management. Infect Dis Rep 2020;12:3-9. doi: 10.4081/idr.2020.8543.

9. Flisiak R, Horban A, Jaroszewicz J, Kozielewicz D, Pawłowska M, Parczewski $\mathrm{M}$, et al. Recommendations of management in SARSCoV-2 infection of the Polish Association of Epidemiologists and Infectiologists. Pol Arch Intern Med 2020. doi: 10.20452/pamw.15270.

10. Calvo C, García López-Hortelano M, de Carlos Vicente JC, Vázquez Martínez JL, Grupo de trabajo de la Asociación Española de Pediatría para el brote de infección por Coronavirus, colaboradores con el Ministerio de Sanidad, Miembros del Grupo de Expertos de la AEP. Recomendaciones sobre el manejo clínico de la infección por el «nuevo coronavirus» SARS-CoV2. Grupo de trabajo de la Asociación Española de Pediatría (AEP). An Pediatr 2020. doi: 10.1016/j. anpedi.2020.02.001.

11. Resolución Ministerial $N^{\circ} 193-2020-M I N S A$. Documento Técnico: Prevención, Diagnóstico y Tratamiento de personas afectadas por COVID-19 en el Perú 2020.
12. Cao B, Wang Y, Wen D, Liu W, Wang J, Fan G, et al. A Trial of Lopinavir-Ritonavir in Adults Hospitalized with Severe Covid-19. N Engl J Med 2020. doi: 10.1056/NEJMoa2001282.

13. International Society of Antimicrobial Chemotherapy. Official Statement from International Society of Antimicrobial Chemotherapy (ISAC) 2020 (acceso 12 abril 2020). Disponible en: https://www.isac.world/ news-and-publications/official-isac-statement.

14. Gautret P, Lagier JC, Parola P, Hoang VT, Meddeb L, Mailhe M, et al. Hydroxychloroquine and azithromycin as a treatment of COVID-19: results of an open-label non-randomized clinical trial. Int J Antimicrob Agents 2020; 20:105949. doi: 10.1016/j.ijantimicag.2020.105949.

15. Parker M, Bhattacharya S, Blanchet K, Caney S, Ying Yang Chan E, da Costa Thomé B, et al. Research in global health emergencies. Nuffield Counc Bioeth 2020 (acceso 12 abril 2020). Disponible en: https://www.nuffieldbioethics.org/publications/research-in-global-health-emergencies.

16. Organización Panamericana de la Salud. Orientación y estrategias para agilizar la revisión y supervisión éticas de investigaciones relacionadas con COVID-192020 (acceso 12 abril 2020). Disponible en: https://www.paho. org/es/documentos/orientacion-estrategias-para-agilizar-revision-supervision-eticas-investigaciones.

17. World Health Organization. "Solidarity" clinical trial for COVID-19 treatments n.d. (acceso 8 abril 2020). Disponible en: https://www.who.int/emergencies/diseases/novel-coronavirus-2019/global-research-on-novel-coronavirus-2019-ncov/solidarity-clinical-trial-for-covid-19-treatments.

18. Liu J, Cao R, Xu M, Wang X, Zhang $\mathrm{H}, \mathrm{Hu} \mathrm{H}$, et al. Hydroxychloroquine, a less toxic derivative of chloroquine, is effective in inhibiting SARS-CoV-2 infection in vitro. Cell Discov 2020;6:1-4. doi: 10.1038/ s41421-020-0156-0.

19. Wang M, Cao R, Zhang L, Yang X, Liu J, Xu M, et al. Remdesivir and chloroquine effectively inhibit the recently emerged novel coronavirus (2019-nCoV) in vitro. Cell Res 2020;30:269-71. doi: 10.1038/s41422020-0282-0.

20. Gao J, Tian Z, Yang X. Breakthrough: Chloroquine phosphate has shown apparent efficacy in treatment of COVID-19 associated pneumonia in clinical studies. Biosci Trends 2020;14:72-3. doi: 10.5582/bst.2020.01047.

21. Touret F, de Lamballerie X. Of chloroquine and COVID-19. Antiviral Res 2020;177. doi: 10.1016/j.antiviral.2020.104762.

22. Nakkazi E. Randomised controlled trial begins for Ebola therapeutics. The Lancet 2018;392:2338. doi: 10.1016/S0140-6736(18)33011-3.

23. Sheahan TP, Sims AC, Graham RL, Menachery VD, Gralinski LE, Case JB, et al. Broad-spectrum antiviral GS-5734 inhibits both epidemic and zoonotic coronaviruses. Sci Transl Med 2017;9:eaal3653. doi: 10.1126/ scitranslmed.aal 3653 . 
24. Holshue ML, DeBolt C, Lindquist S, Lofy KH, Wiesman J, Bruce H, et al. First Case of 2019 Novel Coronavirus in the United States. N Engl J Med 2020;382:929-36. doi: 10.1056/NEJMoa2001191.

25. Mulangu S, Dodd LE, Davey RT, Tshiani Mbaya O, Proschan M, Mukadi D, et al. A Randomized, Controlled Trial of Ebola Virus Disease Therapeutics. NEngl J Med 2019;381:2293-303. doi: 10.1056/NEJMoa1910993.

26. Choy KT, Yin-Lam Wong A, Kaewpreedee P, Sia S-F, Chen D, Yan Hui $\mathrm{KP}$, et al. Remdesivir, lopinavir, emetine, and homoharringtonine inhibit SARS-CoV-2 replication in vitro. Antiviral Res 2020:104786. doi: 10.1016/j.antiviral.2020.104786.

27. Chan JF-W, Yao Y, Yeung M-L, Deng W, Bao L, Jia L, et al. Treatment With Lopinavir/Ritonavir or Interferon- $\beta 1 \mathrm{~b}$ Improves Outcome of MERS-CoV Infection in a Nonhuman Primate Model of Common Marmoset. J Infect Dis 2015;212:1904-13. doi: 10.1093/infdis/jiv392.

28. Murdoch D, Lyseng-Williamson KA. Spotlight on Subcutaneous Recombinant Interferon- $\beta$-1a (Rebif ${ }^{\star}$ ) in Relapsing-Remitting Multiple Sclerosis. BioDrugs 2005;19:323-5. doi: 10.2165/00063030200519050-00005.
29. Arabi YM, Asiri AY, Assiri AM, Aziz Jokhdar HA, Alothman A, Balkhy $\mathrm{HH}$, et al. Treatment of Middle East respiratory syndrome with a combination of lopinavir/ritonavir and interferon- $\beta 1 \mathrm{~b}$ (MIRACLE trial): statistical analysis plan for a recursive two-stage group sequential randomized controlled trial. Trials 2020;21:8. doi: 10.1186/s13063-019-3846-x.

30. Sayburn A. Covid-19: trials of four potential treatments to generate "robust data" of what works. BMJ 2020;368. doi: 10.1136/bmj.m1206.

31. IDL reporteros. El martillo y el baile 2020. La estrategia del gobierno contra el COVID-19, detallada por Víctor Zamora, ministro de Salud (acceso 11 abril 2020). Disponible en: https://idl-reporteros.pe/el-martillo-y-el-baile/.

32. Wu Z, McGoogan JM. Characteristics of and Important Lessons From the Coronavirus Disease 2019 (COVID-19) Outbreak in China: Summary of a Report of 72314 Cases From the Chinese Center for Disease Control and Prevention. JAMA 2020;323:1239-42. doi: 10.1001/jama.2020.2648.

33. Gozzer E, Canchihuamán F, Piazza M, Vásquez H, Hijar G, Velásquez A. Contribución del Perú en las iniciativas para promover la seguridad sanitaria mundial. Rev Peru Med Exp Salud Pública 2016;33:574-9. doi: 10.17843/rpmesp.2016.333.2324. 